\title{
E-Health und Pflege - Chancen für eine Verbesserung der Versorgung ${ }^{1}$
}

\section{MATTHIAS VON \\ SCHWANENFLÜGEL}

Dr. Matthias von Schwanenflügel, ist Leiter der Unterabteilung Haushalt, Recht, Telematik im Bundesministerium für Gesundheit und Lehrbeauftragter an der Universität Münster

\author{
Auch im Bereich der pflegerischen Versorgung \\ müssen weiterhin Maßnahmen mit dem Ziel \\ ergriffen werden, die Effizienz der eingesetzten \\ Ressourcen nachhaltig zu erhöhen. Informations- \\ und Kommunikationstechnologien spielen hierbei \\ eine wesentliche Rolle. Ihre Anwendung im \\ Gesundheitsbereich ist deshalb von Bedeutung, \\ weil sie das Potential haben, die Qualität \\ und Effizienz der Versorgung zu erhöhen \\ und die Rolle der Patienten zu stärken.
}

\section{Problemaufriss}

Die Gesundheitssysteme der Industrieländer sind alle mit ähnlichen Herausforderungen und Problemen konfrontiert: Die demographische Entwicklung, der technische Fortschritt und ihre Einflüsse auf die Versorgungsstrukturen. Die Lebenserwartung steigt weiter ${ }^{2}$ und mit ihr auch die Zahl der Menschen mit Pflegebedarf. Heute beträgt die Zahl der Pflegebedürftigen in Deutschland rund 2,46 Mio. Personen, bis zum Jahr 2050 wird sie sich fast verdoppeln auf rund 4,37 Mio. ${ }^{3}$. Für immer mehr zu versorgende ältere und insbesondere chronisch kranke Menschen stehen mehr spezialisierte Behandler mit immer besseren und differenzierteren technischen Möglichkeiten für Diagnostik und Therapie zur Verfügung. Angesichts dieser Zahlen wird der Druck auf die Erschwinglichkeit von Leistungen des Gesundheitssystems zunehmen. Der steigenden Zahl von älteren und vor allem multimorbiden Menschen steht gegenüber eine sinkende Zahl an potentiell Erwerbstätigen ${ }^{4}$. Es ist unstrittig, dass sich die Finanzierungskosten in Zukunft erhöhen werden und dass der medizinisch-technische Fortschritt daran einen erheblichen Anteil hat ${ }^{5}$, wie groß aber dieser Anteil im Verhältnis zur Alterung der Bevölkerung ist, ist umstritten ${ }^{6}$.

Neben der Langzeitpflege wird auch die Nachfrage nach medizinischer Versorgung steigen. Dies wirft Fragen nach der Verbesserung der Effizienz und Qualität der Versorgung auf und der Ruf nach mehr Case-Management, integrativer Versorgung und Entlassungsmanagement wird lauter ${ }^{7}$. Eine mangelhafte Integration der verschie-

\footnotetext{
1 Überarbeitete Fassung eines Vortrags, den der Autor auf der 2nd International Conference on Evidence-based Policy in Long-term Care am 6.9.2012 an der London School of Economics (LSE) gehalten hat. Er dankt Herrn Dittmar Padeken, Leiter des Referats Technische Fragen der Telematik, Anwendung der Telematik und Frau Dr. Doris Wilke im BMG für wertvolle Anregungen

2 Statistisches Bundesamt (Hrsg.), 12. koordinierte Bevölkerungsvorausberechnung, 2009.

3 Quelle: BMG, Zahlen und Fakten, abrufbar unter: www.bmg.bund.de.

4 Fuchs/Sönlein/Weber, IAB-Kurzbericht 16/2011, vgl. http://doku.iab.de/kurzber/2011/kb1611.pdf.

5 Antwort der BReg. v. 16.7.2012 auf die Kl. Anfrage der Abg. Graf u. a., BT-Drs. 17/10312.

6 Ärzte Zeitung v. 31.8.2012 »Demografie, Es gibt größere Kostentreiber «.

7 Colombo et al, Help Wanted? Providing and Paying for LTC, OECD Health Policy Studies, 2011, S. 311.
} 
denen Leistungserbringer bezogen auf einen Patienten kann zu Versorgungsbrüchen und zu Über-, Unter- und Fehlversorgung führen. Gerade das deutsche Versorgungssystem ist fragmentiert und befördert diese Problematiken. ${ }^{8}$

Vor diesem Hintergrund müssen auch im Bereich der pflegerischen Versorgung weiterhin Maßnahmen ergriffen werden mit dem Ziel, die Effizienz der eingesetzten Ressourcen nachhaltig zu erhöhen ${ }^{9}$. Dies ist der Punkt, an dem die Informations- und Kommunikationstechnologie ins Spiel kommt. Ihre Anwendung im Gesundheitsbereich ist von Bedeutung, da sie das Potential hat, die Qualität und Effizienz der Versorgung zu erhöhen und die Rolle der Patienten zu stärken.

In älterwerdenden Gesellschaften ändern sich die Bedürfnisse bezogen auf die Gesundheitsversorgung. Die eingeschränkte Mobilität der älteren und pflegebedürftigen Menschen beeinträchtigt den Zugang zum Gesundheitssystem und die Behandlungsverläufe bei mulitimorbiden Menschen werden komplexer. In diesen Situationen kann die Telemedizin und die Möglichkeit des gemeinsamen Informationszugangs mit Hilfe von E-Health Anwendungen (vgl. Ziff. 2) die

\section{Telemedizin und E-Health Anwendungen können die Versorgung von älteren und pflegebedürftigen Menschen optimieren.}

\section{Versorgung optimieren.}

Pflegefachkräfte arbeiten in allen Versorgungsbereichen und haben eine wichtige Rolle in der integrierten Versorgung, im Case-Management und Entlassungsmanagement. Die Pflegefachkräfte basieren ihre Arbeit auf Dokumentationen und brauchen daher umfassende Informationen über die $\mathrm{Pa}$ tienten. E-Health kann ihre Arbeit nachhaltig unterstützen.

In den Reformbemühungen der letzten Jahre spielte die Integration der verschiedenen Sektoren zur qualitativen Optimierung eine zentrale Rolle ${ }^{10}$, hierzu gehört auch die Delegation von ärztlichen Leistungen an Pflegekräfte und das Entlassungsmanagement ${ }^{11}$. Bei all diesen Optimierungsansätzen kann E-Health unterstützend wirken.

\section{Lösungsmöglichkeiten mit Hilfe von E-Health}

E-Health ist der übergeordnete Begriff für den Bereich der Werkzeuge der Informations- und Kommunikationstechnologien zur Unterstützung und Verbesserung von Prävention, Diagnose, Behandlung, Überwachung und Management von Gesundheit und Lifestyle. ${ }^{12}$ Politisches Ziel ist, die Qualität und Effizienz der Gesundheitsversorgung zu verbessern und die Patienten zu stärken. Die Kommunikation unter den Leistungserbringern und ihr Wissensmanagement kann z. B. verbessert werden. Es können überflüssige Untersuchungen und Folgekosten vermieden werden und die Qualität und Sicherheit der Arzneimittelverordnung kann erhöht werden. Und schließlich kann die Information gegenüber den Patienten optimiert werden und damit der Patient gestärkt sowie seinen Angehörigen geholfen werden. In der Versorgungskette älterer multimorbider und pflegebedürftiger Patienten können die möglichen Brüche zwischen den einzelnen Sektoren ambulanter oder stationärer Pflegebereich und Krankenhaus, Reha und medizinische Versorgung mit Hilfe von E-Health besser überbrückt werden.

Informations- und Kommunikationstechnologien können mit Hilfe des Aufbaus von sicheren und verlässlichen Verbindungen und einheitlichen Vorgaben und Formaten die Kommunikation zwischen Leistungserbringern, z. B. Ärzten, Krankenhäusern und Pflegeeinrichtungen mit Hilfe von standardisierten Arztbriefen oder auch einheitlichen elektronischen Fallakten oder Patientenakten unterstützen und verbessern. Sie dienen dann der gezielten Kommunikation und auch dem Informationsmanagement und im Bereich der Telemedizin der gezielten Informationsverbreitung. Telemedizin ist die Erbringung konkreter medizinischer Dienstleistungen (Diagnostik und Therapie) in Überwindung räumlicher Entfernungen mit Hilfe von Informations- und Kommunikationstechniken; es ist ein Teil von E-Health. ${ }^{13}$

Die sektorenübergreifende, zeit- und raumunabhängige Verfügbarkeit von relevanten Informationen für die (Weiter-)
Behandlung von Patienten kann durch den Einsatz von Informations- und Kommunikationstechnologien unterstützt werden ${ }^{14}$. Dazu gehören Anwendungen der direkten Kommunikation wie Arztbriefe aber auch elektronische Patientenakten. Für Patienten kann ein positiver Effekt die Vermeidung von Doppeluntersuchungen sein. ${ }^{15}$ E-Health kann ferner die Medikation optimieren und sicherer gestalten, den Behandlungsverlauf und den Pflegeprozess über eine Distanz überwachen ${ }^{16}$ und auch ältere Menschen zu körperlichen Aktivitäten mit sog. Game-Boys animieren. Nach Darstellung der OECD ${ }^{17}$ haben Forschungsarbeiten eine positive Korrelation zwischen Einführung der Technologie, Arbeitszufriedenheit bei den Pflegekräften und Effizienz ergeben. Bereits 35\% der Pflegeeinrichtungen in Deutschland nutzen schon eine IT-gestützte Pflegeplanung. ${ }^{18}$ Integrierte Versorgungsformen sind ohne die Anwendung von E-Health (Telematik) effizient nicht machbar. ${ }^{19}$

8 Siehe hierzu umfassend: Sachverständigenrat für die Konzertierte Aktion im Gesundheitswesen, Bedarfsgerechtigkeit und Wirtschaftlichkeit, Bd. III: Über-, Unter- und Fehlversorgung, 2002.

9 Colombo et al, Help Wanted? Providing and Paying for LTC, OECD Health Policy Studies, 2011, S. 295.

10 Vgl. zur Pflegereform 2008 von Schwanenflügel, Die Zukunft der Pflegeversicherung, Zeitschrift für Rechtspolitik 2008, S. 4; Möwisch/Ruser/von Schwanenflügel, Pflegereform 2008, 2008, S. 7 ff; von Schwanenflügel, Moderne Versorgungsformen im Gesundheitswesen, NZS 2006, S. 285.

11 Hübner, Telematik und Pflege, GMS Med Inform Biom Epidemiol. 2006;2(1):Doc01, S. 3.

12 Vgl. Definition der EU Kommission: http:// ec.europa.eu/health-eu/care_for_me/ehealth/index_en.htm (Stand: 20.9.12); allgemein Diamond/Shirky, Health Information Technology: A Few Years Of Magical Thinking?, Health Affairs, 2008, S. 383.

13 Jedamzik, Exzellenzmedizin und E-Health, 2011, S. 63.

14 Hübner, Telematik und Pflege, GMS Med Inform Biom Epidemiol. 2006;2(1):Doc01, S. 5 .

15 Sachverständigenrat zur Begutachtung der Entwicklung im Gesundheitswesen, Wettbewerb an der Schnittstelle zwischen ambulanter und stationärer Gesundheitsversorgung, 2012, Tz. 172.

16 Jedamzik, Exzellenzmedizin und E-Health 2011, S. 114.

17 Colombo et al, Help Wanted? Providing and Paying for LTC, OECD Health Policy Studies, 2011, S. 306.

18 Güttler et al, Pflegedokumentation mit ITSystemen, 2010, S. 57.

19 Haas, Gesundheitstelematik, 2006, S. 195. 
Über die elektronische Patientenakte nach $\mathbb{2} 291$ a SGB V haben die Patienten die Hoheit über ihre Daten und können Ärzten oder auch Pflegekräften ihres Vertrauens den Zugang ermöglichen. Diese elektronische Akte unterstützt die behandelnden und pflegenden Personen neben ihrer eigenen Dokumentation. Telemedizinische Anwendungen wie Überwachung des medizinischen Verlaufs verbessern die Compliance der Patienten bei der Behandlung und unterstützen ihren Wunsch, solange wie möglich in der eigenen Wohnung verbleiben zu können. Sie können auch den Angehörigen helfen, mit anderen in Kontakt zu bleiben und Informationen sowie Erfahrungen auszutauschen. ${ }^{20}$

Die Möglichkeit der elektronischen mobilen Kommunikation unterstützt die Dokumentationsarbeit der Ärzteschaft in den Krankenhäusern und der Pflegekräfte in den Pflegeeinrichtungen ${ }^{21}$. Moderne Anwendungen ermöglichen es, dass nicht mehr mit den großen Aktenschränken über die Flure der Einrichtungen gefahren werden muss, sondern kleine handliche tragbare Computer über W-Lan die gleiche Arbeit erledigen $^{22}$. In vielen Pflegeheimen existieren heute beispielsweise häufig noch drei Dokumentationen: Die klassische Pflegedokumentation, die Dokumentation in der Verwaltung und soweit vorhanden die Dokumentation des die Einrichtung betreuenden Arztes.

Einem Bericht über das Berliner Pflegeheim Bethanien Sophienhaus zufolge $^{23}$, nutzt dort die Internistin des Heimes ein Telemonitoring-System erfolgreich. Dieses System beinhaltet die Pflegedokumentation mit extra Hinweisen seitens der Pflegekräfte aber auch seitens des Systems an die Ärztin. So kann die Ärztin den Gesundheitszustand insbesondere von kritischen Patienten auch kontrollieren, wenn sie nicht vor Ort ist. Sie wird so über Problemlagen frühzeitig informiert mit der Folge, dass weniger gravierende Infektionen und weniger Krankenhauseinweisungen erfolgen. Es müssen auch weniger externe Notdienste in Anspruch genommen werden, was auch zur Verbesserung der Lebensqualität der Pflegebedürftigen beiträgt.

Bei der Bewältigung der ärztlichen und pflegerischen Versorgung auf dem Land ist E-Health ebenfalls nützlich: Das Projekt »Schwester AGnEs « (»Arztentlastende, gemeindenahe, E-Health- gestützte, systemische Intervention « $)^{24}$ bedient sich der Telemedizin, indem es die Arzthelferinnen und Pflegefachkräfte im Rahmen ihrer Hausbesuche, die sie arztentlastend durchführen, bei ihrer Dokumentationsarbeit mit Hilfe eines mobilen Endgeräts unterstützt. Diese sog. eDokumentation hat zum Ziel, dass gemeinsam (Arzt und Arzthelferin) unabhängig von Raum und Zeit dokumentiert wird. ${ }^{25}$

Schließlich ist noch auf »Ambient Assisted Living « (AAL) hinzuweisen. Darunter werden Konzepte, Produkte und Dienstleistungen verstanden, die neue Technologien und soziales Umfeld miteinander verbinden und verbessern mit dem Ziel, die Lebensqualität der Menschen, insbesondere der älteren Menschen, zu erhöhen und den Erhalt sowie die Förderung der Selbständigkeit. Anwendungsfelder sind die Fern- und Selbstüberwachung und auch die Ferndiagnose und Erinnerungs- und Notruffunktionen sowie praktische Anwendungen des täglichen Lebens wie automatische Herdabschaltungen. ${ }^{26}$

\section{Telematikinfrastruktur und elektronische Gesundheitskarte}

Die Qualität der medizinischen Versorgung hängt wesentlich davon $a b$, dass die Ärzte die für die aktuelle Behandlung notwendigen medizinischen Informationen zeitnah und am Ort des Bedarfs erhalten. Mit der elektronischen Gesundheitskarte und der Telematikinfrastruktur ${ }^{27}$ können Daten in Zukunft elektronisch, sicher und schnell zur Verfügung gestellt und zwischen den Behandlern ausgetauscht werden. Die jetzt von den Krankenkassen ausgegebenen Gesundheitskarten sind dafür der erste Schritt. Sie sind der Schlüssel für einen einheitlichen, praktikablen und sicheren Austausch von medizinischen Daten. Ziel ist es, die Qualität der Versorgung $\mathrm{zu}$ verbessern, Kosten zu senken und die Mitwirkungsrechte der Patienten zu stärken.

Im Jahr 2011 erfolgte die Ausstattung der Krankenhäuser sowie der Arzt- und Zahnarztpraxen mit neuen Kartenlesegeräten. Diese können sowohl die elektronische Gesundheitskarte als auch die Krankenversichertenkarte lesen. Seit
Oktober 2011 geben die Krankenkassen elektronische Gesundheitskarten mit Lichtbild an ihre Versicherten aus. Bis auf einige wenige Krankenkassen haben die Kassen die vom Gesetzgeber ( $\$ 4$ Abs. 6 SGB V) vorgegebene Ausstattungsquote in Höhe von $10 \%$ der Versicherten für 2011 erreicht, sodass Ende 2011 ca. 7 Millionen Versicherte eine elektronische Gesundheitskarte erhalten haben. Da die Krankenkassen mit dem Versorgungsstrukturgesetz verpflichtet wurden, im Jahr 2012 weitere 60 \% ihrer Versicherten mit Gesundheitskarten auszustatten, ist davon auszugehen, dass bis Ende 2012 mindestens 50 Millionen Versicherte über eine Gesundheitskarte verfügen werden.

\section{E-Health in der Pflege trägt enseren Information der en bei und stärkt ihre gei der Behandlung.}

Die Organisationen der Selbstverwaltung haben sich darauf verständigt, neben dem Versichertenstammdatenmanagement auch die Notfalldaten und den sicheren Datenaustausch zwischen Ärzten prioritär umzusetzen. Außerdem wird am Beispiel der elektronischen Fallakte eine standardisierte Schnittstelle zur Sicherheits- und Kommunikationsinfrastruktur für sog. Mehrwertanwendungen realisiert (unter Mehrwertanwendungen sind hier alle Dienste des

20 Colombo et al, Help Wanted? Providing and Paying for LTC, OECD Health Policy Studies, 2011, S. 307.

21 Umfassend Güttler et al, Pflegedokumentation mit IT-Systemen, 2010.

22 Güttler et al, Pflegedokumentation mit ITSystemen, 2010, S. 69.

23 Gießler/Mesic, Telemedizin in der Pflege - eine Chance für mehr Kooperation? Projektarbeit an der Berliner Verwaltungsund Wirtschaftsakademie, Studiengang Gesundheits- und Sozialökonomie (Betreuerin: Reitberger), 2012, S. 126.

24 Vgl. Focus v. 23.3.2009 »Schwester AGnEs nun bundesweit im Einsatz«; Die Welt v. 10.11.2011 »Schwester Agnes auf Hausbesuch $\ll$.

25 Haas, Gesundheitstelematik, 2006, S. 427.

26 Georgieff, Ambient Assisted Living, FAZITSchriftenreihe, 2008, S. 7, 32

27 Sachverständigenrat zur Begutachtung der Entwicklung im Gesundheitswesen, Wettbewerb an der Schnittstelle zwischen ambulanter und stationärer Gesundheitsversorgung, 2012, Tz. 173; Bales/von Schwanenflügel, Die elektronische Gesundheitskarte, NJW 2012, S. 2475. 
Gesundheitssystems zu verstehen, die nicht explizit in $\$ 291$ a SGB V genannt sind). In einer ersten Ausbaustufe werden ein Online-Versichertenstammdatenmanagement sowie die qualifizierte elektronische Signatur als Basis für alle medizinischen Anwendungen getestet und eingeführt. Gleichzeitig müssen die verkammerten Heilberufe (in erste Linie Ärzte und Zahnärzte, aber auch Apotheker und Psychotherapeuten) einen Heilberufsausweis erhalten, der neben der elektronischen Gesundheitskarte der zweite Schlüssel zum System ist. Offizieller Herausgeber dieser Heilberufsweise sind die bei den Ländern angesiedelten Ärzte-, Apotheker- und Psychotherapeutenkammern. Für die anderen Leistungserbringer werden Heilberufsausweise ausgegeben, wenn für diese Berufsgruppen relevante weitere Anwendungen und Dienste eingeführt werden. Die Länder haben sich darauf verständigt für diese nicht verkammerten Berufsgruppen (z. B. Hebammen, Pflegeberufe, Sanitätsfachhändler) ein sog. »elektronisches Gesundheitsberuferegister (eGBR) « einzurichten, das für diese Berufsgruppen die Herausgeberfunktion für die Heilberufsausweise übernimmt. Das eGBR wird seinen Sitz in Nordrhein-Westfalen haben.

Im April 2012 hat die Gematik als Trägerorganisation der Selbstverwaltung für die Telematikinfrastruktur ein Ausschreibungsverfahren für die großflächige Erprobung der ersten Ausbaustufe der Telematikinfrastruktur im Amtsblatt der Europäischen Union veröffentlicht. Die erste Ausbausstufe ermöglicht neben dem bereits erwähnten Versichertenstammdatenmanagement und dem Basisdienst zur Nutzung der qualifizierten elektronischen Signatur für die Leistungserbringern auch die Anbindung von sektorspezifischen Bestandsnetzen und bietet einen sicheren Zugang von der Telematikinfrastruktur zum öffentlichen Internet. Die Erprobung ist Grundlage für die bundesweite Einführung (Online-Rollout).

Im Rahmen des Gesetzes zur Regelung der Entscheidungslösung im Transplantationsgesetz wurden auch die gesetzlichen Grundlagen für die Aufnahme von Erklärungen zur Organspende auf der elektronischen Gesundheitskarte geschaffen. Nach dem gleichen sicherheitstechnischen und datenschutzrechtlichen Prinzip wird es auch möglich sein, andere persönliche Erklärungen des Patienten - z. B. Patientenverfügungen und Vorsorgevollmachten auf der Gesundheitskarte zu speichern und zur Verfügung zu stellen..$^{28}$

\section{Anbindung der Pflege- einrichtungen an die Telematikinfrastruktur}

Im Rahmen des Projekts elektronische Patientenakte ${ }^{29}$ fördert das Bundesministerium für Gesundheit in einem multiprofessionellem Versorgungsszenario Entlassungs-/Überleitungsmanagement mit entsprechender Dokumentation. Das Entlassungsmanagement ist Aufgabe der Krankenhäuser ( $\mathbb{S} 39$ Abs. 1 SGB V) und eine nachhaltige Wahrnehmung dieser Aufgaben beginnt bereits mit der Aufnahme der Versorgung in der Einrichtung. Ein "Überleitungsmanagement « gilt übergreifend für einen Wechsel der Versorgung vom ambulanten zum stationären Bereich und vice versa. Zentrales Element eines "guten Übergangsmanagements" ist die Kommunikation auf unterschiedlichen Ebenen, d. h. mit dem Patienten und seinen Angehörigen, innerhalb des multidisziplinären Behandlungsteams und zwischen den Leistungserbringern

\section{Die elektronische Patienten- und Pflegeakte ist Basis für multidisziplinäre, berufsübergreifende Zusammenarbeit der beteiligten Berufsgruppen.}

Arzneimitteldokumentation, die Notfalldaten und schließlich auch die Patientenverfügung. Durch den Zugriff auf eine gemeinsame Dokumentation soll eine zeitnahe Anpassung der Pflege und der Krankenversorgung ermöglicht werden und existierende Hürden zur Veranlassung notwendiger Interventionen leichter überwunden werden können.

Ziel des Projekts ist es, eine sektorübergreifende Dokumentation für die jeweilige sektorspezifische Verwendung zu erhalten. Dies dient der Herstellung der Kontinuität der Versorgung bei der Übergabe und der Verbesserung der Akutversorgung durch Basisdokumentation (z. B. für das Szenario notdiensthabender Arzt trifft auf unbekannten Patienten) zu gewährleisten. Schließlich sollen die pflegenden Angehörigen bei ihrem Engagement unterstützt werden.

In diesem Kontext wird auch an $\mathrm{Va}$ rianten gearbeitet: So ist die »elektronische Fallakte e ein Beispiel für eine gemeinsame elektronische, krankheitsfallbezogene Dokumentation in Verantwortung der Leistungserbringer (nach einmaliger Zustimmung des Patienten) im Rahmen kooperativer Versorgungsstrukturen (dies kann auch eine Pflegeeinrichtung mitumfassen). Das Projekt »elektronische Patientenakte nach \$291a SGB V« des BMG sieht eine Schnittstelle zwischen der fall- und sektorübergreifenden Akte unter der Datenhoheit des Patienten und der fallspezifischen Akte unter der $\mathrm{Da}$ tenhoheit der beteiligten Leistungserbringer vor - beide Dokumentationsformen ergänzen sich - auf keinen Fall schließen sie sich gegenseitig aus.

der unterschiedlichen Sektoren. Insbesondere bei den pflegebedürftigen, oft multimorbiden älteren Patienten ist ein häufiger Wechsel zwischen ambulanter Pflege, häuslicher und stationärer Krankenversorgung eher die Regel denn die Ausnahme. Die Dokumentationen der ambulanten und stationären Pflege sollen mit denen der ärztlichen Dokumentation zusammengeführt werden. Die elektronische Patienten- und Pflegeakte ist damit Basis und ein wichtiges Verbindungsstück für multidisziplinäre, berufsübergreifende Zusammenarbeit der beteiligten Berufsgruppen; z. B. braucht die Pflege Zugriff auf den Arztbrief, die

\section{Ausblick}

Auf die auf der Gesundheitskarte gespeicherten oder über sie verfügbar gemachten medizinischen Daten (vgl. Ziff. 3) dürfen nur Ärzte, Zahnärzte und Apotheker zugreifen - mit Ausnahme der Notfalldaten und der persönlichen Erklärungen ist hierfür die explizite Einwilligung des Patienten erforderlich. Technisch wird dies durchgesetzt, indem

28 Bales/von Schwanenflügel, Die elektronische Gesundheitskarte, NJW 2012, S. 2475.

29 Hierzu allg. Haas, Gesundheitstelematik, 2006, S. 434. 


\section{Körperschaftsstatus der Krankenkassen}

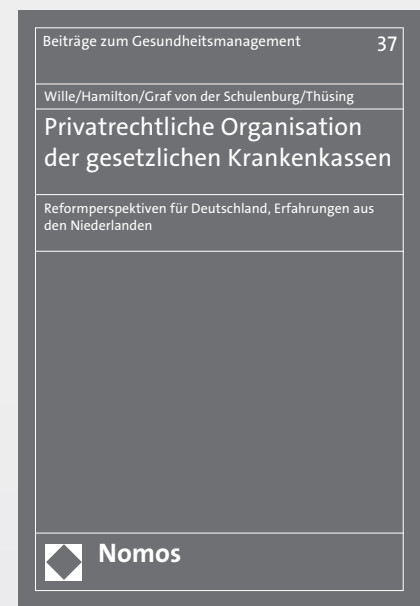

\section{Privatrechtliche Organisation der gesetzlichen Krankenkassen}

Reformperspektiven für Deutschland, Erfahrungen aus den Niederlanden

Von Prof. Dr. Eberhard Wille, Geert Jan Hamilton, Prof. Dr. JohannMatthias Graf von der Schulenburg, Prof. Dr. Gregor Thüsing

2012, 259 S., brosch., 38,-€

ISBN 978-3-8329-7635-4

(Beiträge zum Gesundheitsmanagement, Bd. 37)

Gesetzliche Krankenkassen sind aufgrund ihres Körperschaftsstatus an eine enge Rahmenordnung gebunden, die ihr wettbewerbliches Handeln vorgibt. Das Gutachten analysiert die Fragestellungen, die sich bei der Überführung der Kassen in eine private Rechtsform ergeben würden und bewertet die wettbewerbliche Wirkung sowie die verfassungsrechtliche Zulässigkeit.

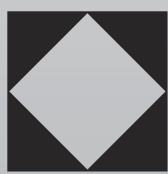

Nomos 